\title{
¿Es significativa la participación \\ de estudiantes de pregrado en las revistas médicas colombianas indexadas?
}

\section{Is the participation of undergraduate students significant to indexed medical journals in Colombia?}

\author{
Carlos Andrés Gualdrón Frías ${ }^{1}$, Jhuliana Mercedes Castillo Cabellos ${ }^{1}$, \\ Laura Tatiana Calderón Nossa ${ }^{1}$
}

El ingreso de un nuevo estudiante a la educación superior implica un reto para la sociedad del conocimiento, que independiente de una formación técnica, persigue lograr el desarrollo integral de los jóvenes profesionales persiguiendo dos objetivos básicos: "enseńar a investigar y hacer investigación", que se refieren al propósito de generar competencias para desarrollar investigación válida y confiable (1).

Si bien la comunidad estudiantil es parte crucial y definitiva en este proceso de creación, también se debe considerar el papel de las directivas y profesorado, pues aunque la publicación científica supone un avance y crecimiento social y personal, también las

\section{Historial del artículo}

Fecha de recepción: 31/08/2018

Fecha de aprobación: 10/06/2019

1 Universidad Pedagógica y Tecnológica de Colombia. Grupo de investigación ACEMED-UPTC. Tunja, Boyacá, Colombia.

Correspondencia: Carlos Andrés Gualdrón Frías. Dirección: Departamento de Medicina, Universidad Pedagógica y Tecnológica de Colombia. Avenida Central del Norte 39-115, Tunja, Boyacá, Teléfono: (57+8) 7405626 Correo Electrónico: carlos.gualdron@uptc.edu.co

Como citar este artículo: Gualdrón-Frías C, Castillo-Mercedes J, Calderón-Laura T. ¿Es significativa la participación de estudiantes de pregrado en las revistas médicas colombianas indexadas?. Revista de la Facultad de Ciencias de la Salud de la Universidad del Cauca. 2019; 21 (2) 38 - 40 
entidades de educación son beneficiadas por estos logros de ciencia, en particular en el campo de la medicina local $(2,3)$; la mayoría de conocimiento nuevo que se presenta en los eventos académicos y profesionales como simposios, congresos y capacitaciones es proveído como resultado de procesos investigativos adelantados en el extranjero (4).

La creación de esta competencia en los futuros médicos es motivada mediante la incentivación de la investigación, fabricando vínculos entre los estudiantes y los procesos investigativos de generación y creación de conocimiento, los cuales se han trazado mediante diversas estrategias como la inclusión de asignaturas específicas en investigación dentro del currículo de la carrera, así como la promoción de diversos grupos externos que persiguen también la creación científica $(5,6)$.

Aunque es bien sabido que la investigación es un escalón esencial en el desarrollo médico estudiantil, en colombia la situación investigativa genera preocupación, y evidencia de esto es que de los 191 artículos originales publicados en la revista colombia médica durante el periodo 1994-2004, 22 artículos (11\%) tuvieron como autores a estudiantes de pregrado y en 14 (63\%) de éstos participaron estudiantes de medicina, lo que es una cantidad relativamente baja en cuanto a la participación estudiantil (5). Así mismo otro estudio en el que analizaron 10 revistas médicas y académicas colombianas indexadas en publindex, de las categorías A1 y A2, en el período 2009-2012 teniendo presentes el número de autores de pregrado por revista, los tipos de publicaciones y el volumen de las mismas, entre otros parámetros, se evidenció que la participación estudiantil fue de 122 (7\%) de un total de 1738 artículos evaluados (7).

En Perú, también pudo apreciarse un bajo grado de participación de los estudiantes de pregrado, que podría reflejar escaso interés por la investigación estudiantil ya que para el ańo 2008, el 4\% del total de publicaciones científicas peruanas contaban con la participación de estudiantes. Merece destacar, que el 75\% de dicho porcentaje correspondió a artículos basados en la participación de investigadores graduados, lo que pone de relieve la importancia del apoyo de investigadores experimentados (8). El estímulo que significó la participación de profesores y/o investigadores en concurrencia con los estudiantes se vio reflejado en los últimos ańos, puesto que se pudo evidenciar un aumento en la producción científica peruana en pregrado, ya que en el período 2009$2010,12.9 \%$ de las publicaciones originales en revistas indizadas en scielo-perú contaban con la participación de un estudiante de medicina como coautor (9).

El artículo científico es parte primordial en el proceso investigativo y la investigación no se encuentra completa mientras los resultados no sean publicados (10). A pesar de que no todos los trabajos llegarán a ser un artículo original, todos tienen el potencial, si son guiados de forma adecuada, para convertirse en una publicación que en un futuro sea fuente de conocimiento aprovechable $(9,11)$.

"La investigación estimula el pensamiento crítico y la creatividad, y en base a estos elementos el proceso de aprendizaje se revitaliza y se combate la memorización, que tanto ha contribuido a formar profesionales pasivos, poco amantes de la innovación, con escasa curiosidad e iniciativa personal" (12).

Ya que la investigación en medicina, tiene tantas bondades en la formación profesional, es importante involucrar a estudiantes en formación en la investigación y en la preparación de los artículos originales que son uno de sus productos relevantes. 


\section{CONFLICTO DE INTERÉS}

Los autores manifiestan no tener ningún conflicto de interés

\section{REFERENCIAS}

1. Restrepo Gómez Bernardo. Investigación formativa e investigación productiva de conocimiento en la universidad. Nómadas. 2003; 18:195-202

2. Calvache JA, Delgado-Noguera M. Editorial: El camino de la Revista Facultad Ciencias de la salud. Rev. Fac. Cienc. Salud Univ. Cauca. 2014; 16(1):7

3. Delgado-noguera m. Editorial: un camino propio para nuestro trabajo académico. Rev. Fac. Cienc. Salud Univ. Cauca. 2014; 16(2):8

4. Calvache JA, Chaparro LE, Chávez A, Delgado MB, Fonseca N, Montes FR, et al. Estrategias y obstáculos para el desarrollo de la investigación en programas de anestesiología: documento de consenso en Colombia. Rev. Colomb. Anestesiol. 2012; 40(4): 256-261

5. Pachajoa-londońo HM. Publicación de artículos originales desde el pregrado en una revista médica colombiana entre 1994-2004. Ciencia e Investigación Médica Estudiantil Latinoamericana. 2006; 11(1):24-26.

6. Universidad del Cauca, C. de F. C. de la S. Editorial: Recomendaciones para la transformación de la educación médica en Colombia. Revista De La Facultad De Ciencias De La Salud De La Universidad Del Cauca. 2017; 19(1), 12-15

7. Granados JPA, Roa MC, Romero DMS, Pulido JC, Guarte HGG. Participación de estudiantes de pregrado de medicina en revistas médicas y académicas colombianas indexadas en Publindex, categorías A1 y A2, en el período 2009-2012: Revisión sistemática de la literatura. Rev Fac Med. 2014; 62(1):9-15

8. Salinas J, Vildózola H. Investigación en pregrado: dificultades y posibles soluciones. Anales de la Facultad de Medicina. 2013; 69(3):216

9. Carrillo-Larco RM, Osada J. Promoción de la investigación: viviendo sin una cultura de publicación. Educ Médica. 2012; 15(3):131-131.

10. Bravo-Peńa M, Barona-Fong L, Campo-López J, Calvache JA. El reporte de la investigación observacional y la declaración STROBE. Rev. Fac. Cienc. Salud univ. Cauca. 2014; 16(3):39-5

11. Gutiérrez C, Mayta P. Publicación desde el pre grado en Latinoamérica: importancia, limitaciones y alternativas de solución. Cimel ciencia e investigación médica estudiantil latinoamericana. 2003:8(1): 54-60.

12. Carvajal Tapia AE. Importancia y reflexiones sobre la investigación y publicación científica desde pregrado. Scientifica. 2014; 12(1). 